\title{
Multi-locus sequence typing of Ehrlichia ruminantium strains from geographically diverse origins and collected in Amblyomma variegatum from Uganda
}

Ryo Nakao', Joseph W Magona ${ }^{1,2}$, Lijia Zhou $^{1}$, Frans Jongejan ${ }^{3,4}$ and Chihiro Sugimoto ${ }^{1 *}$

\begin{abstract}
Background: The rickettsial bacterium Ehrlichia ruminantium is the causative agent of heartwater in ruminants. A better understanding of the population genetics of its different strains is, however, needed for the development of novel diagnostic tools, therapeutics and prevention strategies. Specifically, the development of effective vaccination policies relies on the proper genotyping and characterisation of field isolates. Although multi-locus sequence typing (MLST) has been recently developed, only strains from geographically restricted collections have been analysed so far. The expansion of the MLST database to include global strains with different geographic origins is therefore essential. In this study, we used a panel of reference strains from geographically diverse origins and field samples of E. ruminantium detected from its vector, Amblyomma variegatum, in heartwater-endemic areas in Uganda.

Results: A total of 31 novel alleles (six, four, six, three, two, five, three, and two for gltA, groEL, lepA, lipA, lipB, secY, sodB, and sucA loci, respectively) and 19 novel sequence types (STs) were identified. Both neighbour-joining and minimum spanning tree analyses indicated a high degree of genetic heterogeneity among these strains. No association was observed between genotypes and geographic origins, except for four STs from West African countries. When we performed six different tests for recombination (GeneConv, Bootscan, MaxChi, Chimaera, SiScan, and 3Seq) on concatenated sequences, four possible recombination events were identified in six different STs. All the recombination breakpoints were located near gene borders, indicating the occurrence of intergenic recombination. All four STs that localized to a distinct group in clustering analysis showed evidence of identical recombination events, suggesting that recombination may play a significant role in the diversification of E. ruminantium.

Conclusions: The compilation of MLST data set across the African continent will be particularly valuable for the understanding of the existing genetic diversity of field isolates in African countries. Comprehensive information on the degree of cross-protection between strains and further understanding of possible relationships between genotypes and phenotypes such as vaccine efficacy are expected to lead to the development of region-specific vaccination strategies.
\end{abstract}

\section{Background}

The rickettsial bacterium Ehrlichia ruminantium is the causative agent of heartwater in ruminants, a potential zoonotic disease $[1,2]$ transmitted by ticks of the genus Amblyomma that causes considerable livestock losses in endemic countries [3]. Heartwater is distributed in nearly

\footnotetext{
* Correspondence: sugimoto@czc.hokudai.ac.jp

'Department of Collaboration and Education, Research Center for Zoonosis Control, Hokkaido University, Kita 20, Nishi 10, Kita-ku, Sapporo, Hokkaido 001-0020, Japan

Full list of author information is available at the end of the article
}

all countries of sub-Saharan Africa and has also extended into some islands of the Caribbean, from where it may spread into the American mainland [4]. Evidence from several vaccine trials indicate that a wide range of $E$. ruminantium genotypes with differing cross-protection capacities were simultaneously circulating in the same region $[5,6]$, leading to a poor vaccine efficacy. Therefore, the proper genotyping and characterisation of field isolates of E. ruminantium is an important prerequisite for the development of effective vaccination strategies at regional levels.

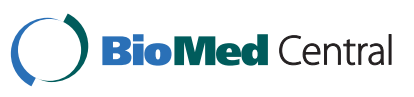

() 2011 Nakao et al; licensee BioMed Central Ltd. This is an Open Access article distributed under the terms of the Creative Commons Attribution License (http://creativecommons.org/licenses/by/2.0), which permits unrestricted use, distribution, and reproduction in any medium, provided the original work is properly cited. 
Several methods have been developed to genotype E. ruminantium. Specifically, typing based on the map1 (major antigenic protein 1) gene has been extensively used and proven to be useful for estimating the genetic diversity of E. ruminantium strains [7-9]. However, these methods are not reliable without proper knowledge of phylogenetic relatedness. Multi-locus sequence typing (MLST) is in turn a powerful typing method that allows determining genetic diversity as well as phylogenetic relationships. Recently, Adakal et al. developed a MLST scheme for E. ruminantium based on eight different housekeeping genes [10]. This method was further evaluated by the same authors and proved to have a resolution high enough to discriminate even between closely related genotypes circulating in Burkina Faso [11]. However, currently available MLST profiles are limited to geographically restricted collections. Considering the wide distribution of E. ruminantium across the African continent, the expansion of the MLST database to include global strains from different geographic origins is therefore needed.

The aim of this study was to examine the MLST method by using a panel of reference strains from geographically diverse origins. Additionally, eight E. ruminantium-positive Amblyomma variegatum collected in Uganda were also investigated to determine the usefulness of this method for the detection of genotypes presently circulating in heartwater-endemic areas. The collection of these data sets is aimed at contributing further to the development of a global database of E. ruminantium genotypes.

\section{Methods}

\section{E. ruminantium reference strains}

The following 14 E. ruminantium strains were sequenced: Ball 3, Burkina Faso, Crystal Springs, Ifé Nigeria, Kerr Seringe, Kiswani, Kwanyanga, Lutale, Pokoase 417, Sankat 430, São Tomé, Senegal, Um Banein, and Zeerust. Their geographic origins and years of isolation are shown in Table 1. All strains were cultured in bovine aorta endothelial cells as described previously [12] and subjected to DNA extraction using the Nucleospin Tissue Kit (Macherey-Nagel, Duren, Germany).

\section{E. ruminantium-positive $A$. variegatum from Uganda}

Eight E. ruminantium-positive tick samples detected by pCS20 PCR [13] were used. Ticks were collected from indigenous cattle from December, 2008 to January, 2009 at heartwater-endemic areas in five different districts in Uganda: Amuria $\left(33.38^{\circ} \mathrm{W}, 02.01^{\circ} \mathrm{N}\right)$, Dokolo $\left(33.10^{\circ} \mathrm{W}\right.$, $\left.01.55^{\circ} \mathrm{N}\right)$, Pallisa $\left(33.42^{\circ} \mathrm{W}, 01.10^{\circ} \mathrm{N}\right)$, Soroti $\left(33.36^{\circ} \mathrm{W}\right.$, $\left.01.43^{\circ} \mathrm{N}\right)$, and Tororo $\left(34.11^{\circ} \mathrm{W}, 00.41^{\circ} \mathrm{N}\right)$. A total of two (A004 and A006), one (D002), two (P003 and P006), two (S001 and S013), and one (T009) samples were collected in Amuria, Dokolo, Pallisa, Soroti, and Tororo, respectively.

\section{Sequencing analysis}

The following eight housekeeping genes were used for DNA sequencing: gltA, groEL, lepA, lipA, lipB, secY, $\operatorname{sod} B$, and $s u c A$. PCR amplification was conducted using the KAPA 2G Robust PCR Kit as reported by Adakal et al. [10]. PCR products were purified with ExoSAP-IT (USB Corporation, Cleveland, $\mathrm{OH}$ ) and sequenced using the BigDye Terminator version 3.1 Cycle Sequencing Kit (Applied Biosystems, Foster City, CA) and an ABI Prism 3130 x genetic analyzer (Applied Biosystems) according to the manufacturer's instructions. The DNA sequences obtained were submitted to the DNA Data Bank of Japan (DDBJ) (http://www.ddbj.nig.ac.jp) under accession numbers AB625780 to AB625787 (gltA), AB625788 to AB625796 (groEL), AB625797 to AB625810 (lepA), AB625811 to AB625824 (lipA), AB625825 to AB625838 (lipB), AB625839 to AB625852 (secY), AB625853 to $\mathrm{AB} 625860(\operatorname{sodB})$, and AB625861 to AB625874 (sucA).

\section{Data analysis}

Sequences were analysed using GENETYX version 9.1 (GENETYX Corporation, Tokyo, Japan) and were trimmed on both the 5' and 3' ends according to the previous report [10]. The Gardel, Welgevonden (Erwe), and Welgevonden (Erwo) sequences were obtained from the GenBank database (GenBank accession numbers: CR925677, CR925678, and CR767821, respectively). Eight genotypes (Strains 2, 331, 469, 623, 629, 630, 668, and 1062) identified in A. variegatum from Burkina Faso [10] were subjected to phylogenetic, cluster, and recombination analyses. A phylogenetic analysis was carried out for concatenated sequences of eight genes using MEGA 4 programme [14]. Allele sequences for each strain were concatenated in the order gltA-sucA-lepAsodB-lipA-secY-lipB-groEL resulting in a final composite length of $3,419 \mathrm{bp}$. The phylogenetic tree was constructed using the neighbour-joining (NJ) method with 1,000 bootstrap replicates. For each MLST locus, an allele number was assigned to each unique variant. Those sequences identical to previously known alleles were assigned the same allele numbers, whereas those that did not match any known allele were given new allele numbers. A sequence type (ST) was assigned to each distinct combination of alleles at the eight MLST loci. A minimum-spanning tree (MST) was generated using BioNumerics software version 6.5 (Applied Maths, Saint-Martens-Latem, Belgium). The Simpson's diversity index (DI) was calculated for each locus to determine its discriminatory power. Alignment files of each gene locus and concatenated sequences of all loci were imported 
Table 1 MLST profiles of E. ruminantium strains from geographically diverse origins and collected in A. variegatum from Uganda

\begin{tabular}{|c|c|c|c|c|c|c|c|c|c|c|}
\hline \multirow[t]{2}{*}{ Strain } & \multirow[t]{2}{*}{ Origin } & \multirow[t]{2}{*}{ Year of isolation } & \multicolumn{8}{|c|}{ Allele } \\
\hline & & & gltA & groEL & lepA & lipA & $\operatorname{lipB}$ & $\sec Y$ & $\operatorname{sod} B$ & sucA \\
\hline Ball 3 & South Africa & $N R^{a}$ & 5 & 6 & 13 & 1 & 5 & 4 & 1 & 9 \\
\hline Burkina Faso & Burkina Faso & 1997 & 1 & $3(4)^{b}$ & 1 & 1 & 12 & 1 & 1 & 1 \\
\hline Crystal Springs & Zimbabwe & 1990 & 6 & 7 & 14 & 6 & 13 & 15 & 9 & 9 \\
\hline Gardel & Guadeloupe, Caribbean & 1982 & 1 & 1 & 3 & 1 & 4 & 1 & 1 & 2 \\
\hline Ifé Nigeria & Nigeria & 1983 & 7 & $3(4)$ & 2 & 1 & 1 & 1 & 1 & 1 \\
\hline Kerr Seringe & The Gambia & 2001 & 3 & 5 & 1 & 3 & $6(7)^{c}$ & 16 & 3 & 5 \\
\hline Kiswani & Kenya & 1985 & 1 & 8 & 3 & 1 & 2 & 17 & 10 & 4 \\
\hline Kwanyanga & South Africa & $N R$ & 8 & $3(4)$ & 15 & 7 & 5 & 15 & 11 & 9 \\
\hline Lutale & Zambia & 1986 & 2 & 6 & 3 & 1 & 5 & 17 & 1 & 4 \\
\hline Pokoase 417 & Ghana & 1996 & 9 & 5 & 3 & 1 & 4 & 1 & 3 & 2 \\
\hline Sankat 430 & Ghana & 1996 & 3 & 5 & 1 & 3 & $6(7)$ & 16 & 3 & 5 \\
\hline São Tomé & São Tomé and Principe & 1981 & 1 & 6 & 16 & 8 & 3 & 18 & 1 & 1 \\
\hline Senegal & Senegal & 1981 & 9 & 5 & 1 & 3 & $6(7)$ & 19 & 3 & 5 \\
\hline Um Banein & Sudan & 1981 & 2 & 6 & 17 & 1 & 5 & 17 & 1 & 4 \\
\hline Welgevonden (Erwe) & South Africa & 1981 & 2 & $3(4)$ & 2 & 1 & 5 & 1 & 1 & 4 \\
\hline Welgevonden (Erwo) & South Africa & 1985 & 2 & $3(4)$ & 2 & 1 & 5 & 4 & 1 & 4 \\
\hline Zeerust & South Africa & 1979 & 10 & 6 & 18 & 1 & 13 & 15 & 1 & 10 \\
\hline \multicolumn{11}{|c|}{ A. variegatum samples from Uganda } \\
\hline $\mathrm{A} 004$ & Amuria, Uganda & 2008-2009 & 1 & 9 & 17 & 1 & 1 & 1 & 1 & 4 \\
\hline A006 & Amuria, Uganda & 2008-2009 & 1 & 9 & 17 & 1 & 1 & 1 & 1 & 4 \\
\hline D002 & Dokolo, Uganda & 2008-2009 & 2 & $3(4)$ & 3 & 1 & 8 & 17 & 1 & 4 \\
\hline P003 & Pallisa, Uganda & 2008-2009 & 1 & $3(4)$ & 17 & 1 & 3 & 17 & 1 & 4 \\
\hline P006 & Pallisa, Uganda & 2008-2009 & 2 & $3(4)$ & 3 & 1 & 8 & 17 & 1 & 4 \\
\hline S001 & Soroti, Uganda & 2008-2009 & 2 & $3(4)$ & 3 & 1 & 8 & 1 & 1 & 4 \\
\hline S013 & Soroti, Uganda & 2008-2009 & 1 & 6 & 2 & 1 & 1 & 1 & 1 & 1 \\
\hline T009 & Tororo, Uganda & 2008-2009 & 1 & 9 & 17 & 1 & 1 & 1 & 1 & 4 \\
\hline No. of alleles & & & 9 & 7 & 9 & 5 & 9 & 7 & 5 & 6 \\
\hline No. of mutated sites per locus & & & 15 & 11 & 10 & 11 & 13 & 24 & 7 & 12 \\
\hline Size $(b p)$ & & & 395 & 447 & 475 & 341 & 358 & 587 & 415 & 401 \\
\hline Polymorphism (\%) & & & 3.80 & 2.46 & 2.11 & 3.23 & 3.63 & 4.09 & 1.69 & 2.99 \\
\hline$D I^{d}$ & & & 80.3 & 80.0 & 85.7 & 42.0 & 88.7 & 78.3 & 47.0 & 73.7 \\
\hline$d N / d S^{e}$ & & & 0.36 & 0.22 & 0.43 & 0.22 & 0.30 & 0.14 & 0.40 & 0.33 \\
\hline
\end{tabular}

${ }^{\mathrm{a}} \mathrm{NR}$, not previously recorded in the literature.

${ }^{\mathrm{b}}$ The sequences of STs 3 and 4 of groEL deposited in GenBank are identical.

'The sequences of STs 6 and 7 of lipB deposited in GanBank are identical.

dDI, Simpson's diversity index.

$e^{e} d N / d S$, the ratio of nonsynonymous to synonymous substitutions.

into SplitsTree4 program version 4.11.3 [15] and a preliminary network was obtained using the neighbour-net algorithm [16]. PHI test was conducted to determine whether recombination events were statistically significant. We further evaluated recombination events using six methods implemented in Recombination Detection Program version 3.44 (RDP3) [17], including GeneConv [18], Bootscan [19], MaxChi [20], Chimaera [21], SiScan [22], and 3Seq [23]. If one sequence was identical to another sequence, only one representative sequence from each group was included in this analysis.

\section{Results and Discussion}

All MLST loci were successfully amplified from the 14 reference strains and eight Ugandan tick samples. The sequencing analysis of the amplified PCR products revealed that 103 nucleotide sites $(3.01 \%)$ were polymorphic in a total of 3,419 bp from the eight MLST loci (Table 1). The lowest number of polymorphisms per locus was seven in the $\operatorname{sod} B$, followed by 10 in lepA, 11 in the groEL and $\operatorname{lip} A, 12$ in the $\operatorname{suc} A, 13$ in the $\operatorname{lip} B, 15$ in the $g l t A$, and 24 in the $\sec Y$ locus. The percentage of polymorphic sites ranged from $1.69 \%$ in $\operatorname{sodB}$ to $4.09 \%$ 
in the $\sec Y$ locus. Since multiple sequences were not detected by direct sequencing of PCR products, we assumed that the sequences obtained from each locus originated from a single population.

$\operatorname{SodB}$ was the most conserved locus among the strains examined in this study, as similarly found for a previous comparison among E. ruminantium-positive tick samples in Burkina Faso [11], indicating that $\operatorname{sodB}$, and specifically E. ruminantium-specific $\operatorname{sodB}$ LAMP [13], is a suitable target for the genetic identification of this species. Conversely, the locus with highest percentage of polymorphic sites was $\sec Y(4.09 \%)$, which is similar to the percentage previously observed for Burkina Faso (4.60\%) [11]. Out of 103 single nucleotide polymorphisms, a total of 22 (21.4\%) resulted in nonsynonymous amino acid changes. The ratios of nonsynonymous to synonymous substitutions $(\mathrm{dN} / \mathrm{dS})$ were $0.36,0.22,0.43,0.22,0.30,0.14,0.40$, and 0.33 for gltA, groEL, lepA, lipA, lipB, secY, sodB, and sucA loci, respectively, indicating an accumulation of synonymous mutations at all loci.

Three sets of strains or tick samples, the pair Kerr Seringe and Sankat 430, the pair D002 and P006, and the triplet A004, A006, and T009, had identical sequences in all eight MLST loci. Although Kerr Seringe and Sankat 430 were from geographically isolated countries (The Gambia and Ghana, respectively [24,25]), a high level of similarity in the sequences from these strains have been previously reported based on the analysis of the highly polymorphic map1 gene [8]. Therefore, using only these target genes might not be sufficient to enable the discrimination between closely related strains.

Allele numbers were assigned to each sequence as indicated in Table 1. A total of 31 novel alleles were identified (six, four, six, three, two, five, three, and two for gltA, groEL, lepA, lipA, lipB, $\sec Y$, $\operatorname{sod} B$, and $\operatorname{suc} A$ loci, respectively). To evaluate the discriminatory power of each locus, DI values were calculated using BioNumerics software. DI ranges from 0 to 100 , namely from a low to a high diversity. The lowest DIs were associated with the lipA (42.0) and $\operatorname{sodB}(47.0)$ loci, suggesting that these genes are the least informative, as the DI values for the other loci were higher than 70.0. This result is also reflected in the identical allelic profiles for the lipA and sod $B$ loci in the eight Ugandan tick samples (Table 1). STs were determined for each reference strain and tick sample based on the alleles identified at each locus. Out of 25 samples (17 reference strains and eight Ugandan tick samples), a total of 21 STs were identified. Except for the STs of the Gardel and Welgevonden (Erwo) strains, which were also included in a previous study [10], 19 STs were novel.

In addition to 17 reference strains and eight Ugandan samples, eight STs previously detected in A. variegatum from Burkina Faso were included for further analyses.
We first performed a cluster analysis based on ST profiles. The resulting MST revealed the existence of three main groups (named I, II, and III) (Figure 1). Group I was the largest, consisting of four reference strains and three Ugandan tick samples. Group II was composed of two reference strains and three samples from Burkina Faso and one sample from Uganda. Group III consisted of three reference strains and two samples from Burkina Faso. Eight reference strains and seven samples, three from Burkina Faso and four from Uganda, were not included in any group. There was no association between groups and geographic origins, except for four STs in group III, all of which originated from West African countries (Burkina Faso, Ghana, Senegal, and The Gambia). A phylogenetic analysis based on a 3,419-bp concatenated sequence of eight genes revealed that these four STs were also clustered together, while others were not clustered according to their geographic origins in a NJ tree (Additional file 1). Therefore, even though MST and NJ analyses rely on distinct analytical principles, both methods suggested a high degree of genetic heterogeneity among the strains examined and highlighted the genetic isolation of STs in group III.

There are several hypotheses that could explain the cause of a lack of association between STs and their geographic origins. For example, the migration of E. ruminantium from other endemic regions, presumably due to transportation of infected animals or ticks, as previously evidenced by the introduction of this pathogen into the Caribbean [26], could be responsible. Another possible cause is the temporal spread in terms of the year of isolation; for example, the Ball 3 strain was isolated prior to 1952 [27], while field samples from Burkina Faso and Uganda were collected in 2007-2008 [10] and 2008-2009, respectively. However, we cannot rule out the possibility that this typing method, particularly when employed with the current set of target loci, is not suitable for tracing geographic origins of bacteria, for example, because of the effect of recombination between different genotypes.

We next conducted a neighbour-net analysis to examine the impact of recombination on each locus separately and on the concatenated sequence of all STs. The resulting graphs based on the alignments of individual loci showed tree-like structures and the PHI test did not detect evidence of intragenic recombination (Additional file 2). However, the graph obtained from an alignment of concatenated sequences showed a network structure (Figure 2), providing the evidence for the genetic divergence among the STs examined and highlighting the influence of recombination events on the evolution of this bacterial species (PHI test on the significance of recombination: $p=0.0)$.

In order to find further evidence for intergenic recombination, we performed six different tests for recombination on 


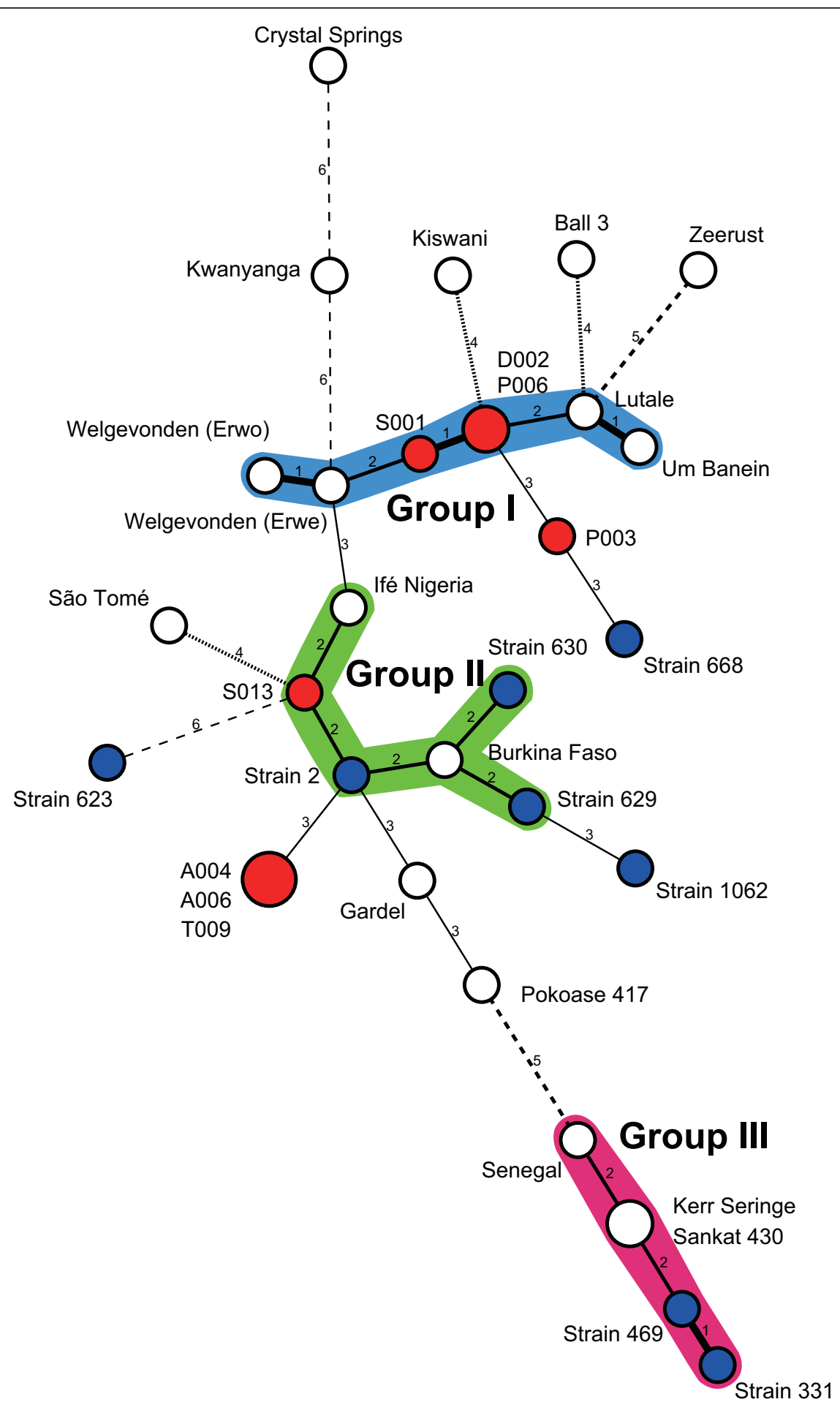

Figure 1 Minimum-spanning tree based on MLST profiles. Each circle in the tree represents a different ST. The white, red, and blue circles represent reference strains, A. variegatum samples from Uganda, and A. variegatum samples from Burkina Faso, respectively. Circle size is proportional to the numbers of strains or tick samples belonging to an ST. Numbers between circles represent the number of allelic differences. Two or more STs differing at less than two loci are regarded as a group and are distinguished by a different colour. The strength of the link (bold, plain, or discontinuous) indicates the degree of genetic similarity (number of common alleles) between STs. 


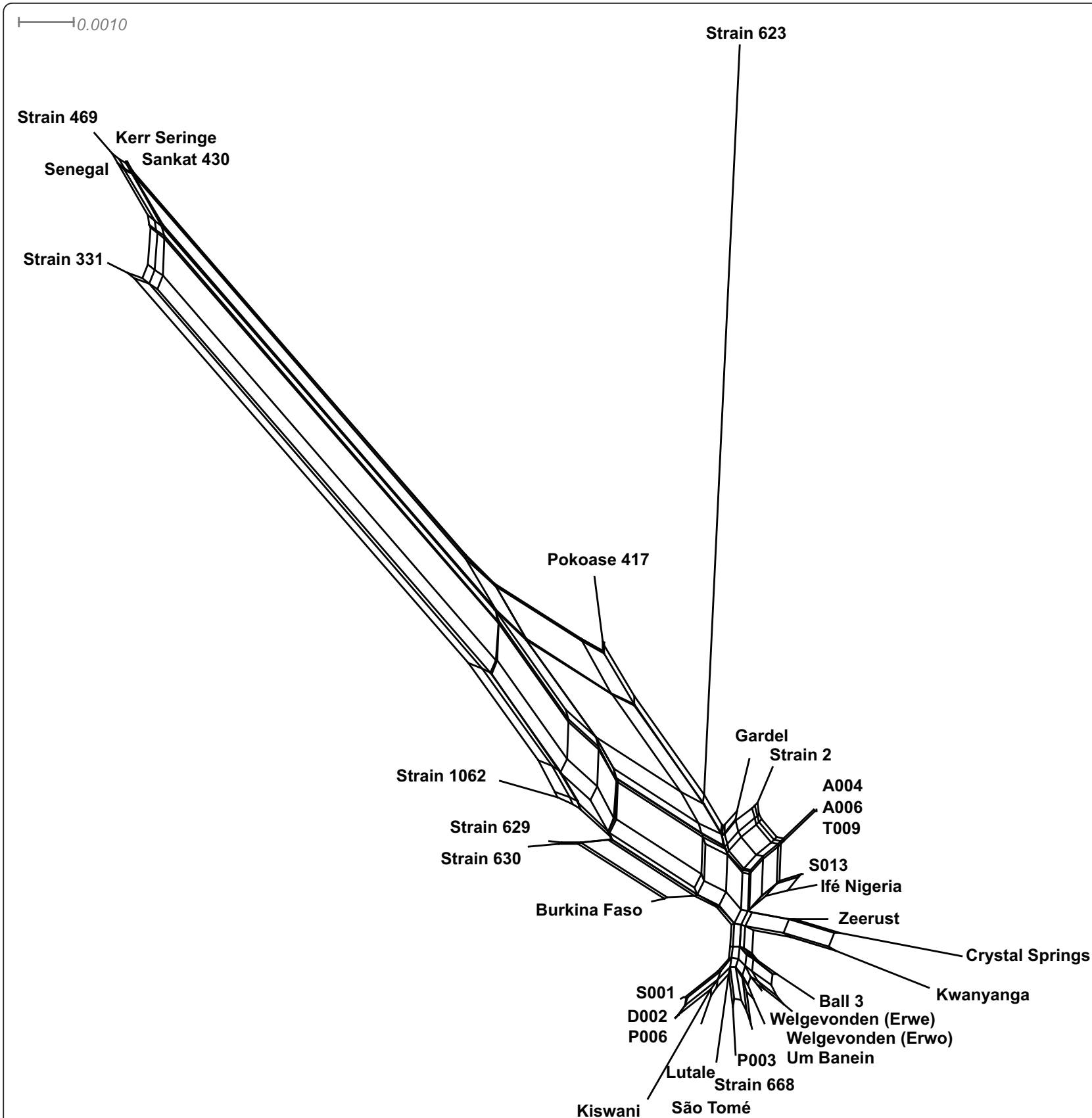

Figure 2 Neighbour-net network constructed from concatenated sequences obtained from all eight loci. The eight housekeeping genes were concatenated in the order gltA-suCA-lepA-sodB-lipA-secY-lipB-groEL. Significant evidence of recombination was obtained by the PHI test $(P=0.0)$.

concatenated sequences. Four possible recombination events were identified in six different STs (Kerr Seringe/ Sankat 430, Senegal, Strains 331, 469, 623, and 1062) (Table 2). A schematic representation of the events is shown in Figure 3A. Events 1 and 2 were supported by all six tests, while events 3 and 4 were supported by three different tests. All the recombination breakpoints were located near gene borders, indicating intergenic recombination.
Strain 1062 from Burkina Faso was predicted to be the result of recombination between S001 from Uganda as a major parent and Kerr Seringe from The Gambia (or Sankat 430 from Ghana) as a minor parent (Table 2). When their sequences were aligned, a clear mosaic pattern was observed (Figure 3B). In addition, A004 (or A006/T009) from Uganda was predicted to be a major parent of a putative recombinant, Strain 623 from Burkina Faso. Considering the geographical 
Table 2 The detection of putative recombination events using six different tests

\begin{tabular}{|c|c|c|c|c|c|c|c|c|c|c|c|}
\hline \multirow[t]{2}{*}{ Event } & \multicolumn{2}{|c|}{ Breakpoint } & \multirow[t]{2}{*}{ Recombinant } & \multirow{2}{*}{$\begin{array}{l}\text { Major } \\
\text { parent }\end{array}$} & \multirow{2}{*}{$\begin{array}{l}\text { Minor } \\
\text { parent }\end{array}$} & \multicolumn{6}{|c|}{ Detection method } \\
\hline & Beginning & Ending & & & & GeneConv & Bootscan & MaxChi & Chimaera & SiScan & 3 Seq \\
\hline 1 & 2965 & 3419 & $\begin{array}{c}\text { Kerr Seringe }{ }^{a} \\
\text { Senegal } \\
\text { Strain } 331 \\
\text { Strain } 469\end{array}$ & Unknown & Ifé Nigeria & + & + & + & + & + & + \\
\hline 2 & 388 & 1284 & Strain 1062 & S001 & Kerr Seringe & + & + & + & + & + & + \\
\hline 3 & 1 & 2651 & $\begin{array}{l}\text { Kerr Seringe } \\
\text { Senegal } \\
\text { Strain } 331 \\
\text { Strain } 469\end{array}$ & Strain 630 & Unknown & + & & & & + & + \\
\hline 4 & 2652 & 3419 & Strain 623 & $\mathrm{~A} 004^{\mathrm{b}}$ & Unknown & & & + & & + & + \\
\hline
\end{tabular}

${ }^{\mathrm{a}} \mathrm{A}$ representative ST of Kerr Seringe and Sankat 430.

${ }^{\mathrm{b}} \mathrm{A}$ representative ST of A004, A006, and T009.

+ , detection of significant recombination $(p<0.05)$.

isolation between major and minor parents of these recombinants (Strains 623 and 1062), this result may support the idea of the existence of a homogeneous population of an ancestral genotype throughout the African continent as previously hypothesised by Adakal et al. [11].
All STs clustered in group III by MST analysis showed evidence of identical recombination events (events 1 and 3 ) (Table 2 and Figure 3A), indicating that recombination may play a significant role in the diversification of E. ruminantium as previously suggested [10,28-30]. In this collection of samples, however, all the STs predicted to be

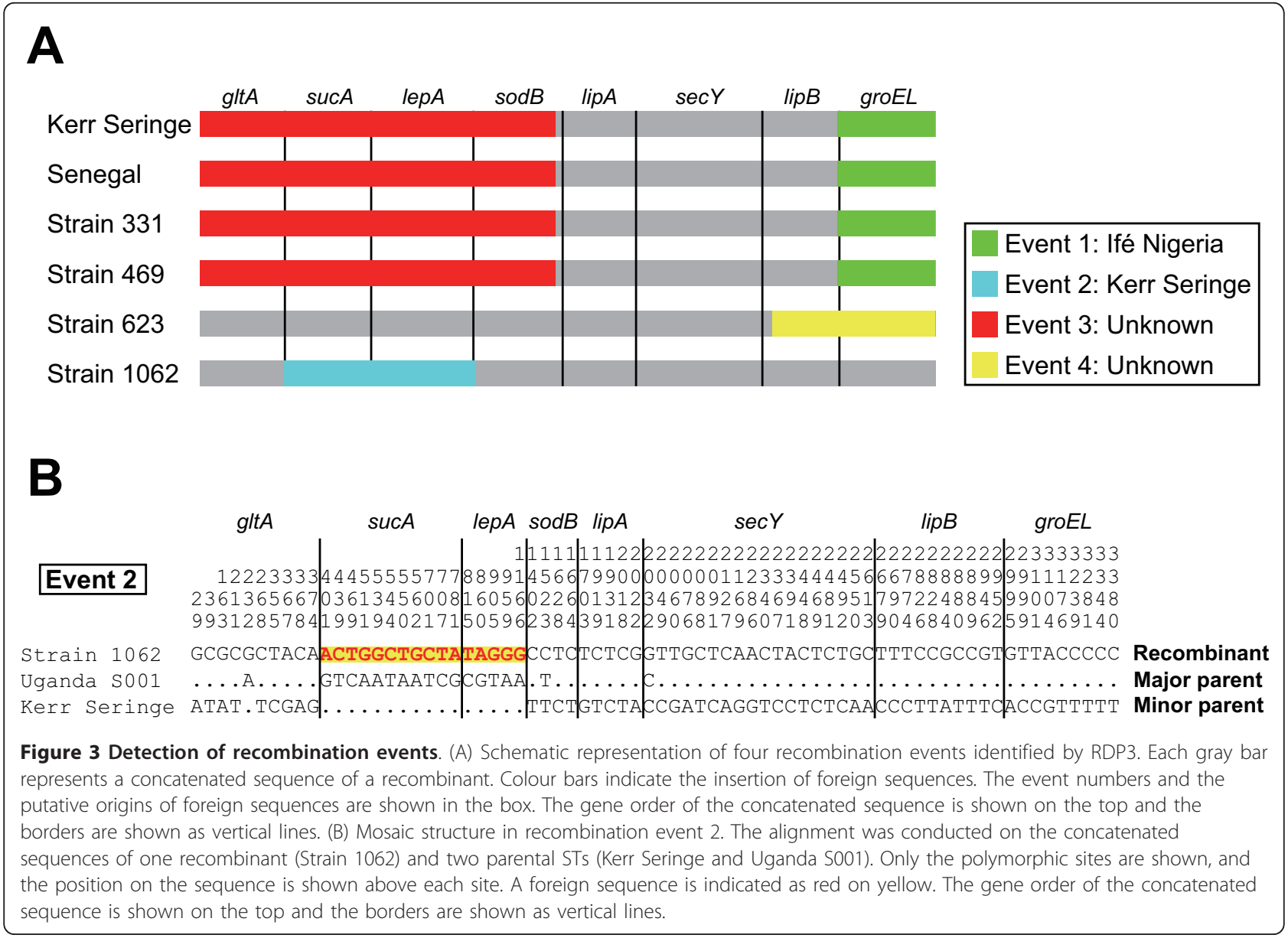


recombinants originated from West African countries. One possible explanation for this regional restriction is that the recombination events could not be properly detected, for example, due to the biased sampling and/or low levels of genetic diversity among the tested strains. Therefore, further compilation of the MLST data, especially of currently circulating isolates in East and Southern African countries, will be invaluable for understanding the role of recombination in bacterial genome evolution and for providing an overview of the current situation of bacterial genetic diversity in African countries.

Finally, the recombination events identified in this study demonstrate that a multi-locus genotyping approach, rather than single-gene based genotyping, is a prerequisite for a proper understanding of phylogenetic relationships of E. ruminantium. The failure to discriminate between two closely related strains, Kerr Seringe and Sankat 430, highlights the need to either improve the MLST method or to develop other multi-locus genotyping methods with higher resolution power, such as the multi-locus variablenumber tandem repeat analysis.

\section{Conclusions}

We investigated a recently developed MLST scheme that allows direct genotyping of E. ruminantium by using global strains from diverse origins and field samples from heartwater-endemic areas in Uganda. As only a limited dataset consisting of geographically restricted isolates was available from previous reports, this study expands the number of allele variants known for each locus. The analyses presented here also provide strong evidence for the occurrence of recombination events among the STs examined. The compilation of MLST data across the African continent will be particularly valuable for understanding the existing genetic diversity of field isolates in African countries. Comprehensive information on the degree of cross-protection between strains and further understanding of possible relationships between genotypes and phenotypes such as vaccine efficacy are expected to lead to the development of region-specific vaccination strategies.

\section{Additional material}

Additional file 1: Neighbour-joining phylogenetic tree based on concatenated sequences obtained from all eight loci. The tree was constructed based on a 3,419-bp concatenated sequence of eight housekeeping genes. One thousand bootstrap replicates were performed for each analysis. Bootstrap values are shown at the nodes.

Additional file 2: Split graph constructed from the sequences of each locus.

\section{Acknowledgements}

The authors would appreciate two anonymous reviewers who considerably helped improve the manuscript. The first author was supported by a research grant fellowship from the Japanese Society for the Promotion of Science (JSPS) for young scientists. This work was supported by Grant-in-Aid for JSPS fellows and for Scientific Research from the Ministry of Education, Culture, Sports, Science and Technology of Japan (MEXT), the program of the Funding Research Center for Emerging and Re-emerging Infectious Disease, MEXT, and the Asia-Africa S \& T Strategic Cooperation Promotion Program from the Special Coordination Funds for Promoting Science \& Technology, MEXT.

\section{Author details}

'Department of Collaboration and Education, Research Center for Zoonosis Control, Hokkaido University, Kita 20, Nishi 10, Kita-ku, Sapporo, Hokkaido 001-0020, Japan. ${ }^{2}$ National Livestock Resources Research Institute (NaLIRRI), P. O. Box 96, Tororo, Uganda. ${ }^{3}$ Utrecht Centre for Tick-borne Diseases (UCTD), Department of Infectious Diseases and Immunology, Faculty of Veterinary Medicine, Utrecht University, Yalelaan 1, 3584CL, Utrecht, The Netherlands. ${ }^{4}$ Department of Veterinary Tropical Diseases, Faculty of Veterinary Science, University of Pretoria, Private Bag X04, Onderstepoort 0110, South Africa.

\section{Authors' contributions}

RN performed PCR and sequencing, conducted data analysis, and draft the manuscript. JWM carried out tick sampling in Uganda. LZ conducted DNA extractions, PCR and sequencing. CS and JF conceived of the study, and participated in its design and coordination and helped to finalize the manuscript. All authors read and approved the final manuscript.

\section{Competing interests}

The authors declare that they have no competing interests.

Received: 15 April 2011 Accepted: 15 July 2011 Published: 15 July 2011

\section{References}

1. Allsopp M, Louw M, Meyer E: Ehrlichia ruminantium: an emerging human pathogen? Ann N Y Acad Sci 2005, 1063:358-360.

2. Louw M, Allsopp M, Meyer E: Ehrlichia ruminantium, an emerging human pathogen-a further report. S Afr Med J 2005, 95:948-950.

3. Mukhebi A, Chamboko T, O'Callaghan C, Peter T, Kruska R, Medley G, Mahan S, Perry B: An assessment of the economic impact of heartwater (Cowdria ruminantium infection) and its control in Zimbabwe. Prev Vet Med 1999, 39:173-189.

4. Burridge $M$, Simmons $L$, Peter $T$, Mahan $S$ : Increasing risks of introduction of heartwater onto the American mainland associated with animal movements. Ann N Y Acad Sci 2002, 969:269-274.

5. Faburay B, Geysen D, Ceesay A, Marcelino I, Alves P, Taoufik A, Postigo M, Bell-Sakyi L, Jongejan F: Immunisation of sheep against heartwater in The Gambia using inactivated and attenuated Ehrlichia ruminantium vaccines. Vaccine 2007, 25:7939-7947.

6. Adakal H, Stachurski F, Konkobo M, Zoungrana S, Meyer D, Pinarello V, Aprelon R, Marcelino I, Alves P, Martinez D, et al: Efficiency of inactivated vaccines against heartwater in Burkina Faso: impact of Ehrlichia ruminantium genetic diversity. Vaccine 2010, 28:4573-4580.

7. Martinez D, Vachiéry N, Stachurski F, Kandassamy $Y$, Raliniaina M, Aprelon R, Gueye A: Nested PCR for detection and genotyping of Ehrlichia ruminantium: use in genetic diversity analysis. Ann N Y Acad Sci 2004, 1026:106-113.

8. Faburay B, Jongejan F, Taoufik A, Ceesay A, Geysen D: Genetic diversity of Ehrlichia ruminantium in Amblyomma variegatum ticks and small ruminants in The Gambia determined by restriction fragment profile analysis. Vet Microbiol 2008, 126:189-199.

9. Raliniaina M, Meyer D, Pinarello V, Sheikboudou C, Emboulé L, Kandassamy Y, Adakal H, Stachurski F, Martinez D, Lefrançois T, et al: Mining the genetic diversity of Ehrlichia ruminantium using map genes family. Vet Parasitol 2010, 167:187-195.

10. Adakal H, Meyer D, Carasco-Lacombe C, Pinarello V, Allègre F, Huber K, Stachurski F, Morand S, Martinez D, Lefrançois T, et al: MLST scheme of Ehrlichia ruminantium: genomic stasis and recombination in strains from Burkina-Faso. Infect Genet Evol 2009, 9:1320-1328.

11. Adakal H, Gavotte L, Stachurski F, Konkobo M, Henri H, Zoungrana S, Huber K, Vachiery N, Martinez D, Morand S, et al: Clonal origin of emerging populations of Ehrlichia ruminantium in Burkina Faso. Infect Genet Evol 2010, 10:903-912. 
12. Jongejan F: Protective immunity to heartwater (Cowdria ruminantium infection) is acquired after vaccination with in vitro-attenuated rickettsiae. Infect Immun 1991, 59:729-731.

13. Nakao R, Stromdahl EY, Magona JW, Faburay B, Namangala B, Malele I, Inoue N, Geysen D, Kajino K, Jongejan F, et al: Development of loopmediated isothermal amplification (LAMP) assays for rapid detection of Ehrlichia ruminantium. BMC Microbiol 2010, 10:296.

14. Tamura K, Dudley J, Nei M, Kumar S: MEGA4: Molecular Evolutionary Genetics Analysis (MEGA) software version 4.0. Mol Biol Evol 2007, 24:1596-1599.

15. Huson DH, Bryant D: Application of phylogenetic networks in evolutionary studies. Mol Biol Evol 2006, 23:254-267.

16. Bryant $D$, Moulton V: Neighbor-net: an agglomerative method for the construction of phylogenetic networks. Mol Biol Evol 2004, 21:255-265.

17. Heath L, van der Walt E, Varsani A, Martin DP: Recombination patterns in aphthoviruses mirror those found in other picornaviruses. J Virol 2006, 80:11827-11832.

18. Padidam M, Sawyer S, Fauquet CM: Possible emergence of new geminiviruses by frequent recombination. Virology 1999, 265:218-225.

19. Martin DP, Posada D, Crandall KA, Williamson C: A modified bootscan algorithm for automated identification of recombinant sequences and recombination breakpoints. AIDS Res Hum Retroviruses 2005, 21:98-102.

20. Smith JM: Analyzing the mosaic structure of genes. J Mol Evol 1992, 34:126-129.

21. Posada D, Crandall KA: Evaluation of methods for detecting recombination from DNA sequences: computer simulations. Proc Natl Acad Sci USA 2001, 98:13757-13762.

22. Gibbs MJ, Armstrong JS, Gibbs AJ: Sister-scanning: a Monte Carlo procedure for assessing signals in recombinant sequences. Bioinformatics 2000, 16:573-582.

23. Boni MF, Posada D, Feldman MW: An exact nonparametric method for inferring mosaic structure in sequence triplets. Genetics 2007, 176:1035-1047.

24. Bell-Sakyi L, Koney EBM, Dogbey O, Abbam JA, Aning KG: Isolation and in vitro cultivation in Ghana of Cowdria ruminantium, the causative agent of heartwater. In Proceedings of the W.A.C.V.A.G.V.M.A. Conference: Accra. Edited by: Koney EBM and Aning KG. Ministry of Food and Agriculture; 1997:46-51.

25. Faburay B, Munstermann S, Geysen D, Bell-Sakyi L, Ceesay A, Bodaan C, Jongejan F: Point seroprevalence survey of Ehrlichia ruminantium infection in small ruminants in The Gambia. Clin Diagn Lab Immunol 2005, 12:508-512.

26. Barré N, Garris G, Camus E: Propagation of the tick Amblyomma variegatum in the Caribbean. Rev Sci Tech 1995, 14:841-855.

27. Haig DA: Note on the use of the white mouse for the transport of strains of heartwater. J S Afr Vet Med Assoc 1952, 23:167-170.

28. Collins N, Liebenberg J, de Villiers E, Brayton K, Louw E, Pretorius A, Faber F, van Heerden $H$, Josemans A, van Kleef M, et al: The genome of the heartwater agent Ehrlichia ruminantium contains multiple tandem repeats of actively variable copy number. Proc Natl Acad Sci USA 2005, 102:838-843.

29. Allsopp M, Allsopp B: Extensive genetic recombination occurs in the field between different genotypes of Ehrlichia ruminantium. Vet Microbiol 2007, 124:58-65.

30. Hughes A, French J: Homologous recombination and the pattern of nucleotide substitution in Ehrlichia ruminantium. Gene 2007, 387:31-37.

doi:10.1186/1756-3305-4-137

Cite this article as: Nakao et al:: Multi-locus sequence typing of Ehrlichia ruminantium strains from geographically diverse origins and collected in Amblyomma variegatum from Uganda. Parasites \& Vectors 2011 4:137.

\section{Submit your next manuscript to BioMed Central and take full advantage of:}

- Convenient online submission

- Thorough peer review

- No space constraints or color figure charges

- Immediate publication on acceptance

- Inclusion in PubMed, CAS, Scopus and Google Scholar

- Research which is freely available for redistribution 